\title{
From Rapid Prototyping to eManufacturing
}

\author{
Nikolai Zaepernick \\ Electo Optical Systems GmbH, Krailling, Germany \\ nikolai.zaepernick@eos.info
}

\begin{abstract}
Additive Manufacturing technologies have been a revolution to R\&D process efficiency and are in place for many years now. The technologies have very much matured ever since and thus seem to pave their way to direct parts business.

Looking back to traditional manufacturing methods, the industrial market has developed highly productive and flexible Machine Tools over time to comply with the requirements of a wide spectrum of industrial applications. These machines have set standards and thus set the expectation benchmark of industry customers.

Additive Manufacturing opens up an enormous additional potential for industrial applications, however competing with traditional production methods. Electro Optical Systems (EOS) is gearing its portfolio and market activities clearly towards manufacturing applications. We will show, which activities EOS are taking to go this road. The example of handling and robotics industry illustrates which additional value Laser Sintering can achieve.

In this context, the gripper technology is a front runner for innovative solutions - lightweight and integrated functionality are key value drivers for handling \& robotics. Existing applications are intensively profiting from this new technology: From gripper weighing less than $20 \mathrm{~g}$ and being able to can handle $10 \mathrm{~kg}$ parts to high-speed pick and place solutions being fully integrated into today's automation environment.

Additive Manufacturing is opening up economically valuable production up to lot size one - metal implant parts for human bodies are another perfect example for this mass individualization approach.
\end{abstract}

Keywords: Digital process chain, rapid prototyping, additive manufacturing, design-driven manufacturing, serial production, plastic Laser Sintering, handling \& robotics, medical implants.

\section{Introduction}

EOS was founded in 1989 and is today the world-leading manufacturer of Laser Sintering systems.

Laser Sintering is the key technology for e-Manufacturing, the fast, flexible and cost-effective production of products, patterns and tools. The technology manufactures parts for every phase of the product life cycle, directly from electronic data. Laser Sintering accelerates product development and optimizes production processes. 
EOS serves customers in more than 50 countries operating sales, application and service offices in 11 countries. With its focus on innovation, EOS reinvests a large portion of its revenues into $\mathrm{R} \& \mathrm{D}$, resulting in a strong patent portfolio with more than 700 active patents in nearly 100 patent families.

EOS completed its business year 2012/2013 with revenues of more than 110 million with a worldwide staff of almost 500 employees, thereof 320 in Germany.

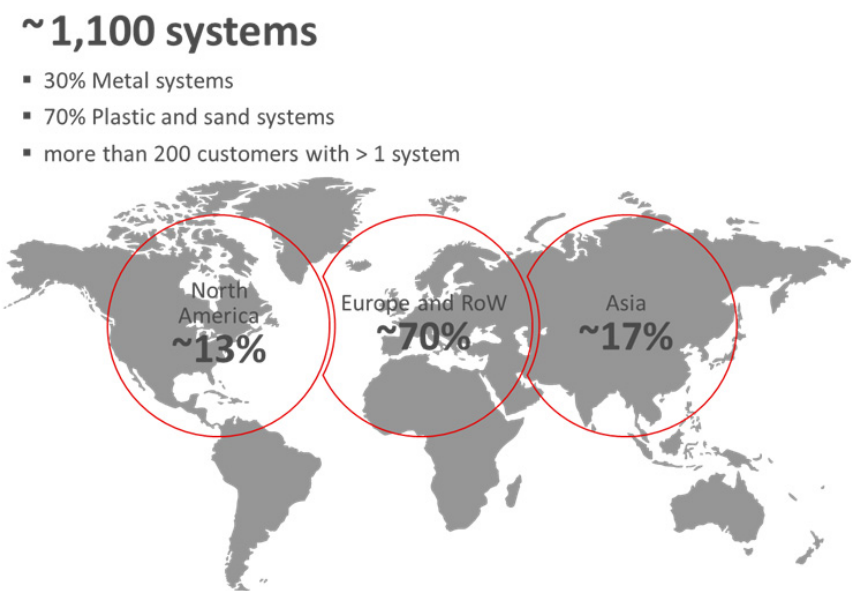

Fig. 1. EOS installed machine base 2012

\section{The History of High Speed Cutting}

High speed cutting today is probably the most exciting example for a production technology in terms of productivity. However this has not always been the case. It took quite a while for milling to get where it is today.

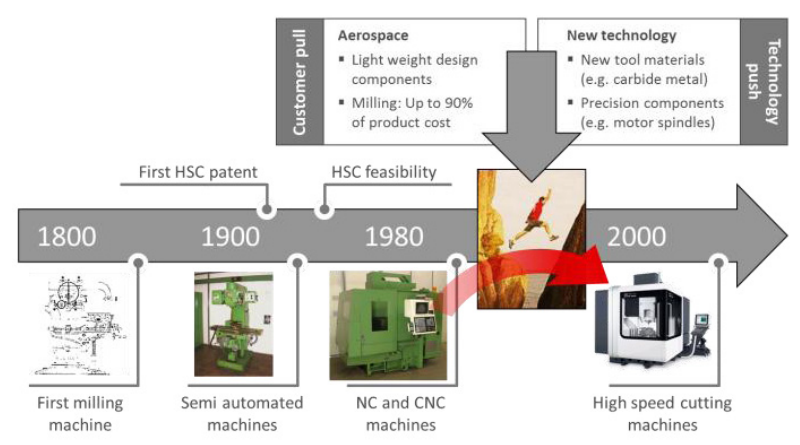

Fig. 2. Schematic development of High Speed Cutting 
It all began in 1818 when Eli Whitney (USA) designed and built the first milling machine for metals. Industrialization has then generated a huge pull for machines tools - manual and semi-automated machines were about to rise. In 1952 J. Parsons constructed the first NC machine using punch tape for machine operation. From the 1970s onwards, CNC machines developed.

Today the High Speed Cutting technology (HSC) is available providing cutting speed over $5.000 \mathrm{~m} / \mathrm{min}$, feed rates factor 10 higher than normal milling and thus $30 \%$ higher material removal rates. Even surface quality is superior at much higher productivity levels. The core question is: 'Why did it take so long for HSC to be established in production'?

Interestingly Dr. Salomon was granted the first HSC patent in 1931 and Lockheed proved HSC feasibility in the 1950s at 10 times the speed (material removal rates $>200$ than actual machines at that time).

It's about 'Crossing the Chasm' as Geoffrey A. Moore describes in his famous book. The technology had to surpass a critical mass before being widely accepted in the market.

Two catalysts were necessary for this development:

- Strong customer pull: Aerospace companies experienced plane ribs to require enormous chipping effort, more than $90 \%$ of total cost related to the part production were related to milling.

- Technology push: New carbide metal tool materials and motor spindles were developed

\section{Readiness of Additive Manufacturing Technologies}

Additive Manufacturing technologies provide high value compared to traditional manufacturing methods. The freedom of design almost makes traditional design obsolete. Engineers can freely design their parts almost without restriction of the production process. Industry experts talk about a 'design-driven manufacturing' process instead. This freedom of design allow for lightweight design as well as very complex geometries.

Furthermore, integrated functionality help to save assembly effort after manufacturing and integrate this production step in the Laser Sintering process itself. This way, hinges and other functional elements can be 'printed in one shot'.

Additive Manufacturing machines make economies of scale obsolete. An economic production of 'lot size one' is no issue any longer. The machine does not care to produce 100 completely different parts or 100 similar parts at the same time. This is the start of mass customization.

Finally, Additive Manufacturing has its roots in the Rapid Prototyping field. A major value proposition is the fact, that machines can produce parts overnight. Time to market has a major influence on $R \& D$ processes and thus open up doors for much more flexible and short term focused product developments. 


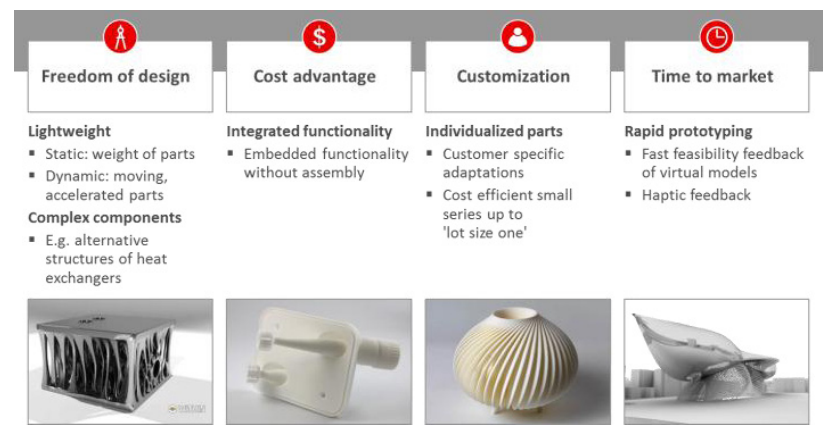

Fig. 3. Key differentiation criteria for Laser Sintering

Are Additive Manufacturing methods yet ready to replace existing production methods like casting or injection molding on the plastic side? At this point in time certainly not - there are some hurdles to be overcome for the broad field of all applications.

Firstly part quality is key and needs to fully comply with customer requirements. This comprises mechanical properties as well as dimensional accuracy and surface quality. With this as prerequisite, AM processes need to be robust - within the build platform, between several jobs, machines and even suppliers. Finally different industries require specific certifications or quality assurance methods and thus also need to be respected.

In many cases, Additive Manufacturing already fulfills these requirements. There are already very interesting applications out there where Additive Manufacturing is a fully accepted mass production method.

And looking ahead on the product roadmaps of AM machine OEMs, the major imitating factors like productivity or build size are being addressed in the near future.
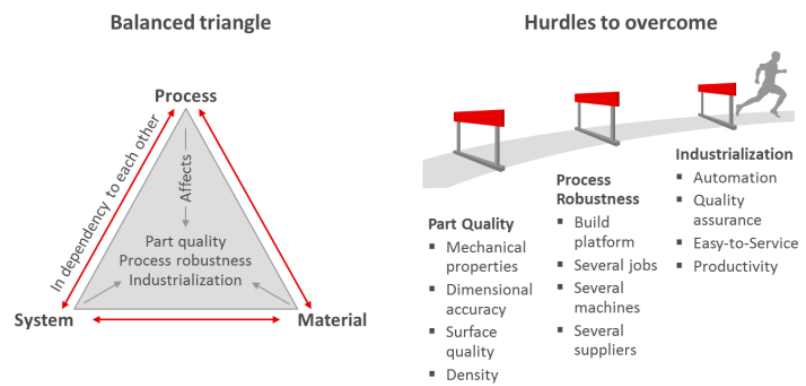

Fig. 4. Implementation hurdles for Additive Manufacturing

\section{$4 \quad$ Application Examples}

For example dental copings and bridges are a 'lot size one' application. Today, there are multiple machines installed to produce 4-5 million individualized metal bridges 
and copings per year. The same applies to drill guides for orthopedic surgery. These plastic parts are produced in the ten thousands area per month fully leveraging the possibilities of Laser Sintering.

Another very promising application lies in the area of handling \& robotics. Lightweight and integrated functionality are the key features making the difference to existing manufacturing methods.

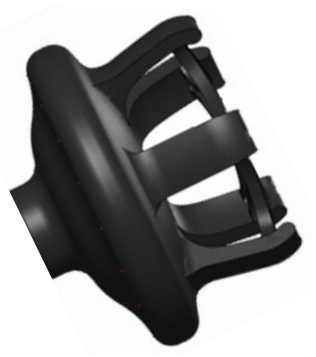

Fig. 5. Example lightweight gripper developed by Kuhn-Stoff

The example of a hole-gripper for part handling purposes recently developed by the company Kuhn-Stoff (www.kuhn-stoff.de) is a perfect one: the gripper weights less than $20 \mathrm{~g}$ and can handle parts up to $10 \mathrm{~kg}$. It operates an integrated pneumatic membrane to apply gripping force.

The advantages of this gripper compared to traditionally manufactured alternatives are

- Weight reduction of about $80 \%$

- Printed in one shot - no final assembly

- Geometry fully flexible and scalable

- Tested to >5 mio. Cycles 